\title{
Monolayer solid of $\mathrm{N}-2 / \mathrm{Ag}(111)$
}

\section{Bruch, L.W.; Hansen, Flemming Yssing}

\section{Published in:}

Physical Review B

Link to article, DOI:

10.1103/PhysRevB.57.9285

Publication date:

1998

\section{Document Version}

Publisher's PDF, also known as Version of record

Link back to DTU Orbit

\section{Citation (APA):}

Bruch, L. W., \& Hansen, F. Y. (1998). Monolayer solid of N-2/Ag(111). Physical Review B, 57(15), 9285-9292. https://doi.org/10.1103/PhysRevB.57.9285

\section{General rights}

Copyright and moral rights for the publications made accessible in the public portal are retained by the authors and/or other copyright owners and it is a condition of accessing publications that users recognise and abide by the legal requirements associated with these rights.

- Users may download and print one copy of any publication from the public portal for the purpose of private study or research.

- You may not further distribute the material or use it for any profit-making activity or commercial gain

- You may freely distribute the URL identifying the publication in the public portal

If you believe that this document breaches copyright please contact us providing details, and we will remove access to the work immediately and investigate your claim. 


\title{
Monolayer solid of $\mathrm{N}_{2} / \mathrm{Ag}(111)$
}

\author{
L. W. Bruch \\ Department of Physics, University of Wisconsin-Madison, Madison, Wisconsin 53706 \\ F. Y. Hansen \\ Department of Chemistry, Technical University of Denmark, IK-207-DTU, DK-2800, Lyngby, Denmark
}

(Received 21 October 1997)

\begin{abstract}
An incommensurate monolayer solid of $\mathrm{N}_{2} / \mathrm{Ag}(111)$ is modeled using extensive molecular-dynamics simulations. The conditions treated range from the low-temperature orientationally ordered solid to the melting of the solid. The properties are evaluated as a function of spreading pressure. Comparison is made to recent experimental data for $\mathrm{N}_{2} / \mathrm{Ag}(111)$ and to results for $\mathrm{N}_{2}$ adsorbed on graphite, $\mathrm{Cu}(110)$, and $\mathrm{MgO}(001)$. [S0163-1829(98)02715-5]
\end{abstract}

\section{INTRODUCTION}

Phenomena reflecting the competing effects of adsorbateadsorbate and adsorbate-substrate interactions are prominent in the physical adsorption of small molecules and have been studied most extensively for nitrogen. Changes in the monolayer relative to the three-dimensional (3D) solid ${ }^{1}$ include (i) differences in the intrinsic geometry from packing in a plane rather than in 3D, (ii) changes in center-of-mass geometry driven by commensurate and near-commensurate lattices of the monolayer on the substrate, and (iii) differences in the character of the superlattice of orientationally ordered molecules. Besides these structural effects, there also are qualitative changes in the sequence of disordering transitions as the temperature is increased, from orientational order to disorder and from solid to dense fluid ("liquid") to dilute fluid ("gas"). In most of the systems studied the effects of (i), (ii), and (iii) are so entwined that it is difficult to appraise them separately, although computer simulations have enabled some comparisons of corrugated and smooth surface models. Adsorption of inert gases on $\mathrm{Ag}(111)$ provided information on systematic trends for the intrinsic 2D solids of monatomic adsorbates, with no apparent effects of the atomic discreteness of the substrate. ${ }^{2}$ Recent measurements ${ }^{3}$ for $\mathrm{N}_{2} / \mathrm{Ag}(111)$ also show no commensurate structures and raise the prospect that comparisons with solids of $\mathrm{N}_{2}$ adsorbed on graphite, $\mathrm{Cu}(110)$, and $\mathrm{MgO}(001)$ may help to separate the effects. The purpose of this paper is to report and discuss computer simulations of the $\mathrm{N}_{2} / \mathrm{Ag}(111)$ system that explore how much of the recent data can be understood with a model of adsorption on a structureless 2D surface and also what phenomena might become accessible with extensions of the experiments.

The phase diagram of monolayer $\mathrm{N}_{2}$ /graphite is the most completely characterized case in molecular physisorption ${ }^{4}$ and presents the remarkable feature that there is no $2 \mathrm{D}$ triple point coexistence of solid, liquid, and gas. The submonolayer commensurate solid melts at $\simeq 48 \mathrm{~K}$, which correlates better ${ }^{5}$ with an estimate of the critical temperature of Lennard-Jones atoms than of the intrinsic 2D triple point temperature of $\simeq 35 \mathrm{~K}$. Apparently, the substrate corrugation stabilizes the solid to higher temperatures than the intrinsic triple point. On an uncorrugated surface, the melting of the monolayer $\mathrm{N}_{2}$ solid might occur at temperatures closer to $35 \mathrm{~K}$. Further, the melting temperature of commensurate $\mathrm{N}_{2}$ /graphite increases from $48 \mathrm{~K}$ to $75 \mathrm{~K}$ as the coverage increases from 0.5 to 1.0 ML; the increase has been discussed in terms of changing mechanisms of vacancy production. At least part of the increase can be attributed to the increase of melting temperature with (2D) spreading pressure, but there has been no systematic analysis of the effect for a molecular solid. This paper includes calculations for smooth $\mathrm{N}_{2}$ /graphite to illustrate the pressure effect and for comparison with smooth $\mathrm{N}_{2} / \mathrm{Ag}(111)$.

The low-temperature solid of monolayer $\mathrm{N}_{2}$ /graphite is orientationally ordered in a two-sublattice herringbone structure and disorders at 26-28 K. The corresponding transition has not been observed ${ }^{3}$ for $\mathrm{N}_{2} / \mathrm{Ag}(111)$, and the question arises as to whether this is because the experiments were not carried to low enough temperatures or because no superlattice is formed of the ordered molecular axes, as appears to be the case for $\mathrm{N}_{2} / \mathrm{MgO}(001)$ (Ref. 6) and $\mathrm{N}_{2} / \mathrm{Cu}(110){ }^{7} \mathrm{~A}$ related issue is "how spherical" the $\mathrm{N}_{2}$ molecule in the orientationally disordered state is and how large a distortion there is from the triangular lattice of spherical molecules. The center-of-mass lattice of $\mathrm{N}_{2} / \mathrm{Ag}(111)$ at $T>25 \mathrm{~K}$ is apparently a triangular lattice, ${ }^{3}$ as for inert gases on $\operatorname{Ag}(111)$, and it is of interest to see the extent to which that is reproduced by a simulation.

The molecular-dynamics simulations reported here are in some ways a simplified version of an extensive set of computations $^{8}$ for commensurate $\mathrm{N}_{2}$ /graphite. Effects of atomic discreteness of the substrate and of electrostatic fields arising from substrate charge distributions are neglected. However, because the center-of-mass lattice is derived from the simulation, rather than input to it, the required volume of computation is very large. Also, the model ${ }^{9}$ of the moleculesubstrate interaction is more primitive than for $\mathrm{N}_{2}$ /graphite because of the absence of adsorption isotherm data and inelastic scattering data.

The organization of this paper is as follows. Section II contains the formulation of the calculations and analysis. Section III contains results for the monolayer solid of 
$\mathrm{N}_{2} / \operatorname{Ag}(111)$ and some results for $\mathrm{N}_{2}$ on flat graphite. Section IV contains a discussion.

\section{INTERACTION MODEL AND SIMULATION METHODS}

The simulations correspond to a smooth surface (no corrugation) version of calculations performed ${ }^{8}$ for $\mathrm{N}_{2} /$ graphite. $^{2}$ Indeed, a parallel series of calculations was performed for the graphite case with the corrugation terms set equal to zero, and the results are discussed in Sec. IV E. In the present work, the $\mathrm{N}_{2}-\mathrm{N}_{2}$ interactions are the same as for $\mathrm{N}_{2}$ /graphite, but the $\mathrm{N}_{2}$-substrate and substrate-mediated dispersion energy terms differ.

\section{A. Molecule-substrate interaction}

The molecule-substrate potential $\mathrm{V}(\mathbf{r})$ is taken to be an atom-atom sum of Morse potentials with parameters set by Tully and co-workers, ${ }^{9}$

$$
\phi(r)=D\left[\exp \left\{-2 \alpha\left(r-r_{0}\right)\right\}-2 \exp \left\{-\alpha\left(r-r_{0}\right)\right\}\right],
$$

with $D=5.2 \mathrm{meV}, r_{0}=3.8 \AA$, and $\alpha=1.2 \AA^{-1}$. The onemolecule adsorption energy (with zero-point energy term) obtained by summing over the top two layers ${ }^{10}$ of the $\mathrm{Ag}(111)$ surface is $79 \mathrm{meV}$, with perpendicular vibration frequency $\omega_{\perp}=4.7 \mathrm{meV}$. The energy/molecule in the quasiharmonic ground state of the monolayer is $96-99 \mathrm{meV}$, depending on which combination of substrate-mediated energy terms is included. For comparison, the experimental heat of adsorption at monolayer condensation is $q_{1}=103 \pm 4 \mathrm{meV} .^{3}$

At low temperatures and low spreading pressures, the modeling of the monolayer phase diagram is insensitive to the precise value of the energy minimum but effects of molecular tipping relative to the surface plane depend on the curvature at the minimum. One piece of information supporting the value of $\omega_{\perp}$ in the $\mathrm{N}_{2} / \mathrm{Ag}(111)$ model is that a scaling of $\omega_{\perp}$ for $\mathrm{N}_{2} /$ graphite $(\sim 6.2 \mathrm{meV}$, from inelastic neutron scattering ${ }^{11}$ ) in analogy to the corresponding values ${ }^{12}$ for adsorbed $\mathrm{Kr}$ gives an estimate of $4.2 \mathrm{meV}$. The $\mathrm{N}_{2} / \mathrm{Ag}(111)$ model used here has the property that tipping of the $\mathrm{N}_{2}$ axis relative to the surface plane requires less energy for $\mathrm{Ag}(111)$ than for graphite. However, there has been a suggestion, ${ }^{13}$ based on electron energy-loss spectroscopy, that the frequency should be in the range 6-9 $\mathrm{meV}$ and hence that the ordering is reversed. Inelastic helium scattering from the $\mathrm{N}_{2} / \mathrm{Ag}(111)$ could determine $\omega_{\perp}$ and provide a basis for an improved model.

For the monolayer simulations the uncorrugated version of this model is used. Only the laterally averaged holding potential $^{14} V_{0}(z)$ is retained. This approximation is made both because of the absence of registry structures in the experiments ${ }^{3}$ and because the use of atom-atom sums to estimate the potential energy corrugation for a monolayer on a close-packed metal surface is known to be unreliable, ${ }^{14,2}$ The lattice constants in the LEED experiment for $\mathrm{N}_{2} / \mathrm{Ag}(111)$ have a large misfit relative to low-order commensurate structures, similar to the case of $\mathrm{Kr} / \mathrm{Ag}(111)$, which has been modeled with a smooth substrate.
The parameters of the substrate-mediated (McLachlan) dispersion energy $^{2}$ are $C_{s 1}=46.4$ au and $C_{s 2}=32.0$ au and the overlayer distance is taken relative to the ideal jellium edge, $L_{o v}=z-(d / 2)$ with $d=2.36 \AA$ for $\operatorname{Ag}(111)$. Effects of dielectric screening of the static $\mathrm{N}_{2}$ quadrupole moment ("static image terms") are neglected.

\section{B. Computations}

The calculations are performed with an isothermal (constrained) molecular-dynamics algorithm ${ }^{15}$ in which the N-N bond distance is fixed at its equilibrium value of $1.098 \AA$. Periodic boundary conditions in directions parallel to the surface plane are used and there are 224 molecules in the monolayer film. This is a classical mechanics approximation and is applied at temperatures down to $15 \mathrm{~K}$; a quasiharmonic lattice dynamics approximation ${ }^{16}$ is used to estimate the size of quantum corrections at the lowest temperatures. The molecular-dynamics technique is essential for treating the experimental conditions of a molecular solid with disordered azimuthal angle of the $\mathrm{N}-\mathrm{N}$ bond. The low temperature orientationally ordered solid requires a more extensive search for the minimum-energy structure than for commensurate $\mathrm{N}_{2}$ /graphite. In both the molecular-dynamics and the quasiharmonic calculations, the search is made in the context of a rectangular unit cell $[=14 \times 16$ molecules in the simulation] and yields two-sublattice herringbone ordering. The sides of the unit cell have ratio $L_{1} / L_{2}=\sqrt{3}$ when the center-of-mass lattice is a triangular lattice. On a smooth surface, the aspect ratio of the herringbone cell differs from $\sqrt{3}$ and is $5 \%$ smaller at the lowest temperature $(15 \mathrm{~K})$ in this work. The main indicator for the 2D solid is the static structure factor evaluated at wave vectors corresponding to the average center-of-mass lattice.

The spreading pressure in the molecular dynamics work is evaluated from the virial theorem ${ }^{17}$ for separate dilations of the two sides of the rectangular cell. Two diagonal elements of the stress tensor $\phi_{x x}$ and $\phi_{y y}$ are evaluated and provide a diagnostic of a trend to herringbone ordering. In the orientationally disordered solid, where the molecular projection on the plane is approximately a disk, one expects $\phi_{x x} \simeq \phi_{y y}$ in a triangular lattice. At low temperatures, the triangular centerof-mass lattice with orientational ordering has $\phi_{x x} \neq \phi_{y y} .{ }^{18}$ Departures from equality of the diagonal components of the stress tensor are used to estimate the extent to which the equilibrium lattice might be distorted from triangular. The search for an unconstrained monolayer solid becomes a matter of adjusting the cell sides $L_{1}$ and $L_{2}$ until $\phi_{x x}=\phi_{y y}=\bar{\phi}$ $=0$.

\section{Thermodynamic characterizations}

The isothermal bulk modulus of the monolayer can be obtained from measurements along an isotherm,

$$
B_{T}=-\left.A \frac{\partial \bar{\phi}}{\partial A}\right|_{T},
$$

for area/molecule $A$. It can also be derived ${ }^{2}$ from measured isobaric thermal expansion coefficients ${ }^{3}$ and the isosteric heat $q_{s t}$ using 


$$
B_{T}=q_{s t} /\left[\left.\sqrt{3} L(\partial L / \partial T)\right|_{p}\right]
$$

where $L$ is the lattice constant of a triangular solid. A comparison of such empirical values with values derived from the simulations provides a rough test of the equation of state of the monolayer solid.

Maxwell relations provided a consistency test for the calculations. The isothermal derivative of the internal energy is

$$
\left.\frac{\partial E}{\partial A}\right|_{T}=\left.T \frac{\partial \bar{\phi}}{\partial T}\right|_{A}-\bar{\phi}
$$

Thus $E$ need not be a monotonic function of $A$. The virial pressures and their temperature derivatives were consistent with the location of zeroes of $\left.(\partial E / \partial A)\right|_{T}$.

The form of phase transition phenomena in these simulations ${ }^{19}$ corresponds to those in an $N-A-T$ ensemble. For instance, the evolution of the system along an isochore with increasing temperature is from (i) solid to (ii) solidliquid coexistence along the melting line to (iii) liquid. The spreading pressure as a function of temperature has three distinct approximately linear segments along such an isochore, with slopes $S_{1}, S_{2}$, and $S_{3}$ respectively, as in Fig. 1(a). The slopes are related by $S_{3}<S_{1}<S_{2}$. Confirmation of the inferred one- and two-phase character of these regimes is provided by molecular trajectory plots. Correlating the twophase segments for different isochores leads to an estimate of the slope of the melting curve $d \phi /\left.d T\right|_{\text {melt }}=\Delta S / \Delta A$. Then, using the bulk modulus, the difference $\Delta A$ in molar areas of liquid and solid at coexistence is obtained, and hence the entropy change $\Delta S$ at melting can be estimated. As in most 2D simulations, ${ }^{2}$ the melting appears to be first order.

The orientational order-disorder transition of $\mathrm{N}_{2}$ /graphite and the corresponding $\alpha-\beta$ transition of 3D $\mathrm{N}_{2}$ are both weakly first order. ${ }^{1,4}$ In these simulations, the structure factor of the leading herringbone diffraction line decreases to zero as the temperature increases from 15 to about $20 \mathrm{~K}$. However, the precision in the calculations is insufficient to decide whether the transition is first or second order. The cell-side lengths are incremented by about $1 \%$ in the searches. The discontinuity in molar volume for $3 \mathrm{D} \mathrm{N} \mathrm{N}_{2}$ is ${ }^{1} 1.4 \%$, equivalent to about $1 \%$ in area. To make a definitive assignment of the order of the transition will require a finer grid of cell sizes, more thorough averaging of fluctuations (longer simulation times) and larger simulation cells. The present state of the interaction model does not appear to warrant such a large computational effort.

\section{MONOLAYER SOLID}

The calculations were done for models denoted $X 1 M$ and $X 1$, respectively, that included or omitted the McLachlan energy; $X 1$ is the designation of the $3 \mathrm{D} \mathrm{N}_{2}-\mathrm{N}_{2}$ pair potential. The mechanism of the substrate-mediated dispersion force should apply for molecules as it does for inert gases. ${ }^{2}$ However, the results here show, in accord with experience for $\mathrm{N}_{2}$ /graphite ${ }^{5,20}$ and for $\mathrm{O}_{2} /$ graphite,${ }^{21}$ that the $X 1$ model follows the experimental lattice constants and the domain of existence of the 2D solid ${ }^{3}$ somewhat better.

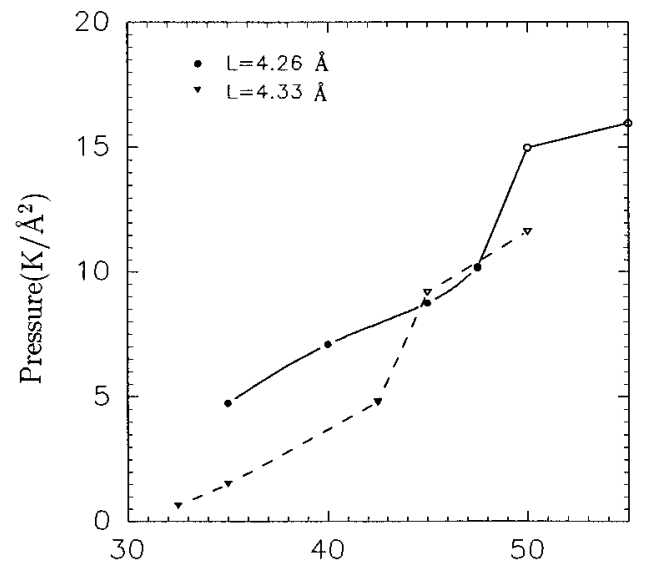

(a) $\mathrm{T}(\mathrm{K})$

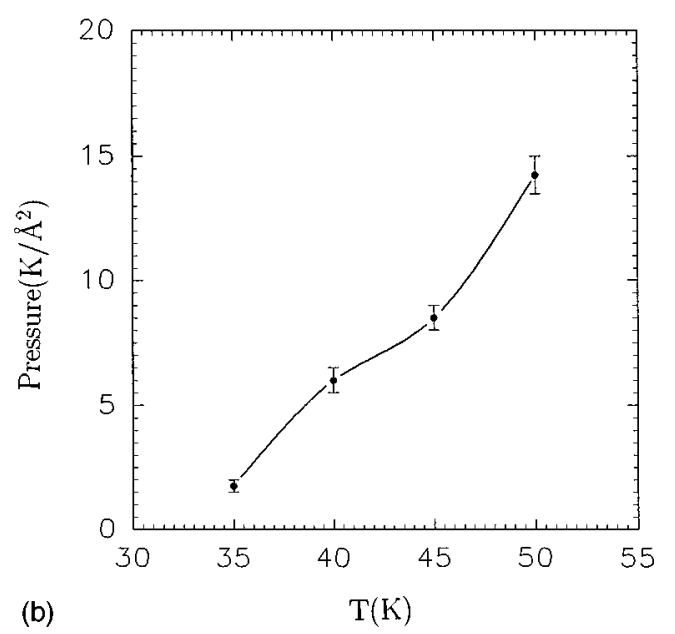

FIG. 1. Spreading pressure as a function of temperature for two isochores and the melting transition. $\bar{\phi}$ (in $\mathrm{K} / \AA^{2}$ ) is shown as a function of $\mathrm{T}$ (in $\mathrm{K}$ ) for the $X 1 M$ model of $\mathrm{N}_{2} / \mathrm{Ag}(111)$. (a) Isochores corresponding to triangular lattices with $L$ equal to $4.26 \AA$ and $4.33 \AA$; filled symbols denote states in which the film is primarily crystalline, open symbols states in which the film is fluid. (b) Melting pressure as a function of temperature obtained from the onset of van der Waals loops in isotherms such as those shown in Fig. 2(b). The lines between plotted points are included only to guide the eye and contain no information beyond the data shown.

\section{A. Benchmarks}

Solutions at $15 \mathrm{~K}$ for the $X 1 M$ solid at zero spreading pressure can be compared for a measure of the contributions of quantum effects and thermally activated disorder. The molecular-dynamics solution is classical and has a rectangular cell $7.1 \times 4.36 \AA^{2}$ with average energy -1142 $\mathrm{K} /$ molecule and area $15.5 \AA^{2} /$ molecule. The $\hbar \rightarrow 0$ (classical) limit of the quasiharmonic approximation has a rectangular cell $7.09 \times 4.44 \AA^{2}$ with average energy $-1149 \mathrm{~K} /$ molecule and $15.8 \AA^{2} /$ molecule. The cell for the quasiharmonic approximation with quantum corrections is dilated by $2 \%$ relative to the $\hbar \rightarrow 0$ structure and is a rectangular cell $7.23 \times 4.51 \AA^{2}$ with $16.3 \AA^{2} /$ molecule. The partial thermal orientational disorder present in the moleculardynamics solution leads to an aspect ratio $L_{1} / L_{2}=0.94 \sqrt{3}$ that is a little larger than for the quasiharmonic approximation. ${ }^{22}$ Overall, the use of a classical molecular 
TABLE I. Isothermal bulk modulus for monolayer solids of $\mathrm{N}_{2}$, in $\mathrm{K} / \AA^{2}$ at specified temperature $\mathrm{T}$ in Kelvin, area $\mathrm{A}$ in $\AA^{2} /$ molecule and spreading pressure $\bar{\phi}$ in $\mathrm{K} / \AA^{2}$.

\begin{tabular}{lcccccc}
\hline \hline Model $^{\mathrm{a}}$ & $T$ & $A$ & $f^{\mathrm{b}}$ & $L^{\mathrm{c}}$ & $\bar{\phi}$ & $B_{T}$ \\
\hline $\mathrm{Ag}(111) / X 1 M$ & 15 & 15.5 & 0.94 & 4.16 & 0 & 200 \\
& 17.5 & 15.5 & 0.96 & 4.19 & 0 & 188 \\
& 20 & 15.6 & 1 & 4.24 & 0 & 135 \\
& $35-40$ & 14.8 & 1 & 4.13 & $\sim 15$ & 195 \\
& $35-40$ & 14.6 & 1 & 4.10 & $\sim 19$ & 230 \\
$\mathrm{Ag}(111) / X 1$ & 50 & $16.0^{\mathrm{e}}$ & & & $\sim 12.5$ & 82 \\
& 15 & 15.2 & 0.94 & 4.12 & 0 & 250 \\
& 17.5 & 15.2 & 0.95 & 4.14 & 0 & 190 \\
& 20 & 15.2 & 0.99 & 4.18 & 0 & 160 \\
$\mathrm{Graphite} / X 1 M^{\mathrm{d}}$ & $35-40$ & 14.8 & 1 & 4.13 & $\sim 11$ & 200 \\
& $35-40$ & 14.6 & 1 & 4.10 & $\sim 15$ & 220 \\
$\mathrm{Ag}(111)^{\mathrm{f}}$ & $35-40$ & 14.8 & 1 & 4.13 & $\sim 23$ & 240 \\
$\mathrm{BN}$ & $35-40$ & 14.6 & 1 & 4.10 & $\sim 28$ & 260 \\
$\mathrm{MgO}(001)^{\mathrm{h}}$ & 60 & $16.0^{\mathrm{e}}$ & & & $\sim 20.5$ & 105 \\
\hline \hline $\mathrm{Subste}$ & $35-40$ & 14.6 & 1 & 4.10 & & 250 \\
& $\sim 60$ & $\sim 16$ & & & & 280 \\
\hline
\end{tabular}

${ }^{a}$ Substrate and notation whether model includes the McLachlan energy term $(X 1 M)$ or omits it $(X 1)$. The precision of calculated $\mathrm{B}_{T}$ is $\sim 10 \%$.

${ }^{\mathrm{b}}$ The aspect ratio of the rectangular unit cell is written as $L_{1} / L_{2}=f \sqrt{3} ; f=1$ denotes a triangular center-ofmass lattice. The area/mol is $A=f \sqrt{3} L_{2}^{2} / 2$.

${ }^{\mathrm{c}}$ Nearest-neighbor center-of-mass spacing in $\AA$. For the rectangular lattices, this spacing is $0.5 \sqrt{L_{1}^{2}+L_{2}^{2}}$.

${ }^{\mathrm{d} C}$ Calculation with flat graphite, retaining only the lateral average $V_{0}$ of the holding potential.

${ }^{\mathrm{e}}$ Calculations for dense fluid at the stated average density.

${ }^{\mathrm{f}}$ Derived from LEED isobars of triangular monolayer solid, Ref. 3, assuming $q_{s t}=103 \mathrm{meV}$.

${ }^{\mathrm{g}}$ From adsorption isotherms for $\mathrm{N}_{2} / \mathrm{BN}$, Ref. 24 near $60 \mathrm{~K}$; identification of density based on x-ray diffraction at $47 \mathrm{~K}$ (Ref. 25).

${ }^{\mathrm{h}}$ From LEED isobars for uniaxially compressed solids of $\mathrm{N}_{2} / \mathrm{MgO}(001)$, Ref. 23.

dynamics simulation seems to be a good approximation down to $20 \mathrm{~K}$ and to still be informative at $15 \mathrm{~K}$.

The structures observed ${ }^{3}$ for $\mathrm{N}_{2} / \mathrm{Ag}(111)$ have area/ molecule in the range $13.9-15.0 \AA^{2}$. In other physisorbed $\mathrm{N}_{2}$ systems it is: $15.7 \AA^{2}$ for commensurate $\mathrm{N}_{2}$ /graphite (and $14.1 \AA^{2}$ in the densest monolayer solid), $14.45 \AA^{2}$ in a higher-order commensurate lattice ${ }^{7}$ of $\mathrm{N}_{2} / \mathrm{Cu}(110)$ and $12.8-14.4 \AA^{2}$ for $\mathrm{N}_{2} / \mathrm{Mg}(001) .^{6}$

The chemical potential increase from monolayer condensation to bilayer condensation along an experimental isobar shown in Ref. 3 is $\approx 300 \mathrm{~K}$. The spreading pressures for the $X 1$ model are consistent with compression of the monolayer to $\approx 4.05 \AA$ at $35 \mathrm{~K}$ without bilayer condensation, using the $\bar{\phi} A$ term of the enthalpy. The chemical potential balance for stability at the smallest observed lattice constants is more problematic for the $X 1 M$ model.

\section{B. 2D Bulk modulus}

Table I contains the bulk moduli of monolayer $\mathrm{N}_{2}$ for several thermodynamic states. Values calculated for the $X 1 M$ and $X 1$ models are presented, as are empirical values derived from isobars for $\mathrm{N}_{2} / \mathrm{Ag}(111)$ (Ref. 3) and $\mathrm{N}_{2} / \mathrm{MgO}(001)$ (Ref. 23) and isotherms for $\mathrm{N}_{2} /$ BN. $^{24}$ Fluctuations in the molecular-dynamics averages lead to $10 \%$ uncertainties in the calculated $B_{T}$.
The calculations at $A=14.6 \AA^{2}$ (triangular lattice with $L$ $\simeq 4.10 \AA$ ) are in good agreement with the bulk modulus derived from the LEED experiments, which probably has $10 \%$ uncertainty in the identification of the relevant isosteric heat. Although the calculated bulk modulus depends primarily on the $\mathrm{N}_{2}-\mathrm{N}_{2}$ interactions, and only weakly on the $\mathrm{N}_{2}$-Ag potential, the differences between the $X 1 M$ and $X 1$ cases are not large enough for the experimental $B_{T}$ values to be a strong discriminator between the two models. The bulk modulus is smaller (compressibility is larger) for the fluid states as a consequence of the lower density.

The similarity of the experimental bulk moduli of $\mathrm{N}_{2}$ on several substrates reflects the transferability of the moleculemolecule interactions in physical adsorption.

The calculated spreading pressures for the monolayer solids above $25 \mathrm{~K}$ appear to be isotropic. That is, $\phi_{x x}=\phi_{y y}$ $\pm 0.2 \mathrm{~K} / \AA^{2}$ in a series of triangular lattices, where the variation appears to be statistical fluctuations in the moleculardynamic averaging. If the increment is assigned to a change in the aspect ratio of the cell from a triangular to a centeredrectangular lattice by uniaxial dilation, there would be a fractional change $\Delta L / L \sim 1 \times 10^{-3}$ along that axis. Thus, the orientationally disordered monolayer molecular solid has a triangular center-of-mass lattice to $0.1 \%$ tolerance. To tighten the limits further is not feasible at the present level of computation. 


\section{Orientationally ordered solid}

The low-temperature solids of $3 \mathrm{D} \mathrm{N}_{2}$ and of monolayer $\mathrm{N}_{2}$ /graphite are orientationally ordered with transition temperatures of $35.6 \mathrm{~K}$ and $\sim 28 \mathrm{~K}$, respectively. ${ }^{4}$ No such transition was observed for $\mathrm{N}_{2} / \mathrm{Ag}(111)$ down to ${ }^{3} 25 \mathrm{~K}$. No systematic extinction of superlattice diffraction spots is seen ${ }^{7}$ for $\mathrm{N}_{2} / \mathrm{Cu}(110)$ in the temperature range $17-32 \mathrm{~K}$. No herringbone order is seen ${ }^{6}$ for $\mathrm{N}_{2} / \mathrm{MgO}(001)$ down to $10 \mathrm{~K}$. The absence of an orientational phase transition in the latter two examples may be a consequence of the frustration of herringbone ordering in unit cells with an odd number of molecules. Thus, the question arises what the character of the axially ordered low-temperature state of $\mathrm{N}_{2} / \mathrm{Ag}(111)$ may be.

The simulations on rectangular cells of 224 molecules show two-sublattice herringbone ${ }^{26}$ ordering in the lowtemperature solid; parameters of the $\bar{\phi}=0$ structure are given in Table I. The structure has " 2 in' ordering, with molecular axes parallel to the plane, and is manifest in a nonvanishing superlattice structure factor ${ }^{5} S(1,2)$. The superlattice strength $S(1,2)$ goes to zero with increasing temperature. There is an inflection point in the plot of quadrupole energy per molecule at about the temperature where $S(1,2)$ vanishes. The transition temperature is between 17.5 and $20 \mathrm{~K}$ for the $X 1 M$ model and just slightly above $20 \mathrm{~K}$ for the $X 1$ model $^{27}$ As the temperature is increased towards the transition, the distribution of molecular tilt angles broadens, indicating that the mechanism for disordering of the molecular axes is similar to that seen in simulations of $\mathrm{N}_{2}$ /graphite. $^{15}$

The orientationally ordered solid has a nontriangular lattice of the molecular centers of mass, with the departure from the $\sqrt{3}$ aspect ratio increasing with increasing order and lowered temperatures. Thus, in addition to the superlattice diffraction spots there should be a structural signature from the lattice distortion.

\section{2D sublimation curve and triple point}

Another reference state is the monolayer at zero spreading pressure, the "unconstrained solid." 2 This is expected to be a good approximation to the state of the solid at monolayer condensation below the $2 \mathrm{D}$ triple point. The calculated lattice constant of the triangular lattice at $\bar{\phi}=0$, derived from isotherms such as those shown in Fig. 2(a), is listed for temperatures from $20 \mathrm{~K}$ to $T_{t}(2 \mathrm{D})$ in Table II. Trajectory calculations confirm that $\mathrm{N}_{2}$ on a smooth graphite or $\mathrm{Ag}(111)$ surface is a normal triple point system with solid, dense fluid (liquid), and dilute (gaseous) phases.

The estimated $T_{t}(2 \mathrm{D})$ of $\mathrm{N}_{2}$ obtained from scaling the 3D triple-point $63 \mathrm{~K}$ (Ref. 1) by the ratio $(0.4 / 0.7)$ of the triple point temperatures of $2 \mathrm{D}$ and $3 \mathrm{D}$ Lennard-Jones atoms ${ }^{2}$ is 36 $\mathrm{K}$. The calculated triple point of $\mathrm{N}_{2} / \mathrm{Ag}(111)$ for the $X 1$ model is $35-37.5 \mathrm{~K}$, and is close to this estimate. For the $X 1 M$ model, the melting temperature is lowered by $5-7 \mathrm{~K}$, on the scale of the decrement found in calculations for commensurate $\mathrm{N}_{2}$ /graphite. ${ }^{5}$

The LEED experiments reported in Ref. 3 tracked (3D) isobars with decreasing temperature, and 2D solid was observed already at temperatures above $38 \mathrm{~K}$. The largest observed lattice constants were $L \simeq 4.15 \AA$. However, the re-
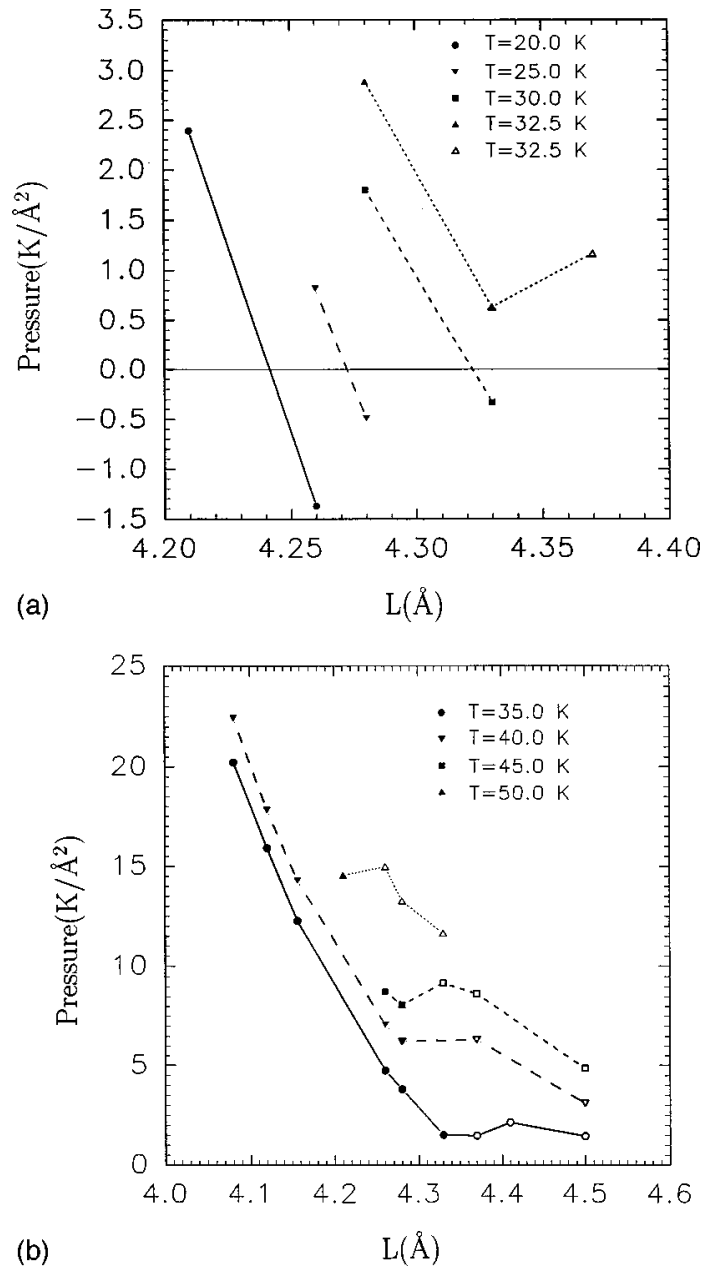

FIG. 2. Isotherms for the $X 1 M$ model. The spreading pressure is plotted as a function of the equivalent triangular lattice spacing $L$. Filled symbols denote crystalline film states while open symbols denote fluid phase points. (a) Isotherms used to determine the zeropressure lattices and the thermal expansion of the unconstrained solid. (b) Isotherms showing van der Waals loops and used to determine the melting-point curve in Fig. 1(b).

sults in Table I indicate that at such $L$ values the system is under considerable spreading pressure. Also, the melting temperature variation for submonolayer $\mathrm{N}_{2}$ /graphite indicates that a lattice with $L=4.26 \AA$ is already under appreciable compressive stress. We therefore conjecture that the experiments crossed the melting curve of $\mathrm{N}_{2} / \mathrm{Ag}(111)$, rather than its sublimation curve. One way to test this would be to determine structures of submonolayer solids of $\mathrm{N}_{2} / \mathrm{Ag}(111)$ at $T<30 \mathrm{~K}$.

These calculations imply that the melting of the monolayer solid is accessible to LEED experiments on $\mathrm{N}_{2} / \mathrm{Ag}(111)$. This may be surprising considering that the melting of inert gas solids on $\mathrm{Ag}(111)$ occurs at temperatures and 3D pressures too high for LEED experiments. However, comparing the ratio of adsorbate-adsorbate potential minimum to the holding potential minimum on silver shows that the scaled lateral interactions are significantly weaker for $\mathrm{N}_{2}$ : $\epsilon / V_{0}$ is 0.11 and 0.16 for $\mathrm{N}_{2}$ and $\mathrm{Kr}$, respectively.

\section{E. 2D melting curve}

The melting transition is identified in isochores and isotherms, which both show signatures of a first-order phase 
TABLE II. Lattice constant of triangular monolayer solid of $\mathrm{N}_{2} / \mathrm{Ag}(111)$ at zero spreading pressure. Nearest-neighbor spacing $L$ in $\AA$ and temperature $T$ in $\mathrm{K} . L(X 1 M)$ and $L(X 1)$ denote values calculated with and without the McLachlan dispersion energy, respectively. The corresponding areas/mol are denoted $A(X 1 M)$ and $A(X 1)$, in $\AA^{2}$.

\begin{tabular}{lcccc}
\hline \hline \multicolumn{1}{c}{$T$} & $L(X 1 M)$ & $A(X 1 M)$ & $L(X 1)$ & $A(X 1)$ \\
\hline 20 & 4.24 & 15.6 & 4.18 & 15.1 \\
25 & 4.27 & 15.8 & 4.21 & 15.3 \\
30 & 4.32 & 16.2 & 4.24 & 15.6 \\
32.5 & $4.35^{\mathrm{a}}$ & 16.4 & & \\
35 & & & 4.27 & 15.8 \\
37.5 & & & $4.30^{\mathrm{b}}$ & 16.0 \\
\hline \hline
\end{tabular}

${ }^{a}$ Metastable solid; simulation shows formation of liquid at run times exceeding 500 ps. The unconstrained solid with $L \approx 4.33 \AA$ melts at $30-32.5 \mathrm{~K}$.

${ }^{\mathrm{b}}$ Metastable solid; simulation shows formation of liquid at run times exceeding 500 ps. The unconstrained solid with $L \approx 4.28 \AA$ melts at $35-37.5 \mathrm{~K}$.

transition. Along an isochore, as described in Sec. II C, the pressure appears to be piecewise linear as a function of temperature. Along isotherms, van der Waals loops appear once the triple-point temperature is exceeded. Some structures were metastable, with equilibration times of longer than 500 ps in the simulation. Figure 1 shows two isochores, with breaks in slope identified as melting transitions, and the melting curve inferred from the isotherms of Fig. 2. There are also breaks in slope at melting for the average height as a function of temperature. Examining the distribution of heights above the surface at a given coverage shows that this break in slope is correlated with the promotion of a few molecules to a second layer, as in simulations for corrugated $\mathrm{N}_{2}$ /graphite, ${ }^{15}$ and that below the melting transition there is a strong broadening of the heights for first layer molecules indicating tipping of molecular axes out of the plane.

\section{Monolayer on $\mathrm{Ag}(111)$}

The triple-point temperature determined by the onset of a van der Waals loop at nearly zero $\bar{\phi}$ is bracketed by 30 $<T_{t}<32.5 \mathrm{~K}$ and $35<T_{t}<37.5 \mathrm{~K}$ for the $X 1 M$ and $X 1$ models, respectively. Although the experiments were carried to $40 \mathrm{~K}$ without a sign of the melting transition, this may be because the thermodynamic path corresponded to significant spreading pressure on the monolayer solid. The higher range for $T_{t}$ in the second case is less at odds with the experiments.

The pressure dependence along two isochores, for $L$ $=4.26$ and $L=4.33 \AA$, is shown in Fig. 1(a). For the $L$ $=4.26 \AA$ case, the melting temperature is in the range $45-50$ $\mathrm{K}$. Direct simulations at $47.5 \mathrm{~K}$ gave a structure factor that was $15 \%$ lower than at $45 \mathrm{~K}$ but still one to two orders of magnitude larger than in the liquid phase at $50 \mathrm{~K}$. This indicated that there might be coexisting regions of solid and liquid; confirmation of the interpretation was given by the trajectory plot shown in Fig. 3. If the limits of the two-phase region are taken to be at 47.5 and $50 \mathrm{~K}$, the difference in area per molecule of the coexisting phases is $\Delta A / A \approx 5 \%$ and the entropy difference is $\Delta S \approx 1.3 k_{B}$. These values are in the range found in simulations of the melting of $2 \mathrm{D}$ inert gases. ${ }^{2}$ The corresponding transition for the $X 1$ model is in the range $50-55 \mathrm{~K}$.

The $4.33 \AA$ isochore showed several instances of metastable structures: solid and fluid phases were found to persist for hundreds of ps. The melting temperature is $\approx 40 \mathrm{~K}$; at

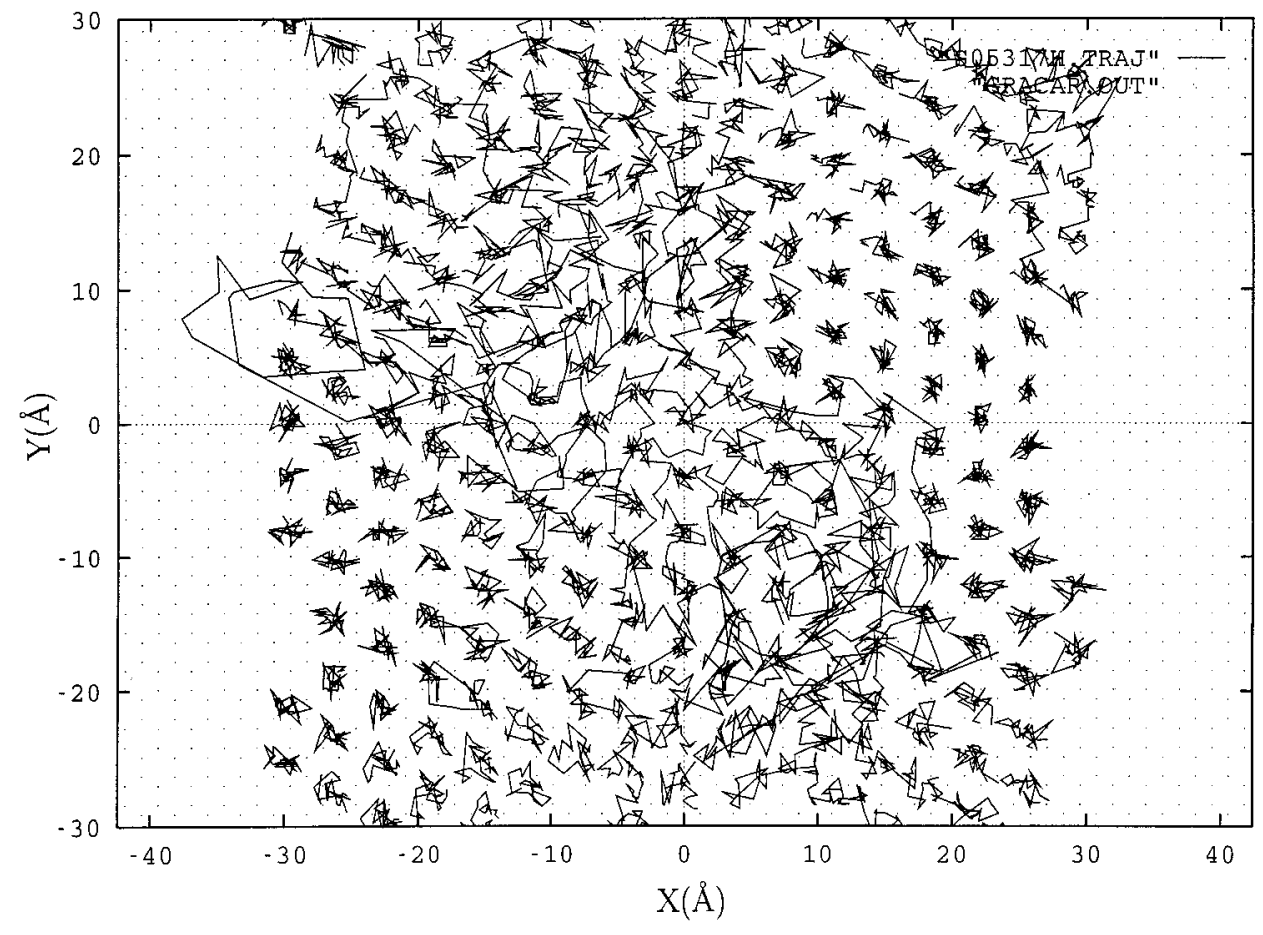

FIG. 3. Trajectory plot for the $X 1 M$ model at $47.5 \mathrm{~K}$ and with density corresponding to a triangular lattice with $L=4.26 \AA$. The trajectories of the $\mathrm{N}_{2}$ centers of mass are shown for 100 ps following 800 ps equilibration time. Coexisting regions of crystalline solid and of fluid are present. The lines connect center-of-mass positions every 4.5 ps in the 100-ps time block. 
$42.5 \mathrm{~K}$ a trajectory plot showed disordered regions with mobile molecules, but some hexagonal ordering remained visible in those regions. The $4.33 \AA$ lattice appears to be beyond the stability limit for the $X 1$ model.

With either of the interaction models, the conclusion is that if the LEED experiments can be carried to $40 \mathrm{~K}$, without too high a 3D gas pressure, the monolayer melting should be evident in the equivalent of a single crystal geometry rather than a powder pattern.

\section{Monolayer on flat graphite}

The case of monolayer $\mathrm{N}_{2}$ on flat graphite was treated as a complement to previous studies of the melting of submonolayer commensurate $\mathrm{N}_{2} /$ graphite $^{8}$ and for comparison with the present $\mathrm{N}_{2} / \mathrm{Ag}(111)$ modeling. The following discussion is for the corresponding $X 1 M$ model. ${ }^{28}$

Using the onset of a van der Waals loop at nearly zero pressure as the criterion, the triple point temperature is bracketed by $27.5<T_{t}<30 \mathrm{~K}$. The calculated melting temperature for 0.5 commensurate monolayer of $\mathrm{N}_{2}$ /graphite is $\sim 34 \mathrm{~K},{ }^{5}$ so the substrate corrugation does stabilize the solid to higher temperatures. The McLachlan energy for $\mathrm{N}_{2}$ /graphite is only $10 \%$ larger than for $\mathrm{N}_{2} / \mathrm{Ag}(111)$, at given density, so that differences in tipping of molecular axes out of the monolayer plane may have some effect on the melting.

The melting temperature for 1.0 commensurate monolayer with this model is $\sim 73 \mathrm{~K} .{ }^{15}$ On flat graphite, a triangular lattice with $L=4.26 \AA$ is under significant compression, as the unconstrained layer at $27.5 \mathrm{~K}$ has $L_{u}=4.36 \AA$. The melting transition of the $4.26 \AA$ isochore of flat graphite occurs at $55-60 \mathrm{~K}$ and $\bar{\phi} \approx 20 \mathrm{~K} / \AA^{2}$. That is, $60 \%$ of the temperature increase from 0.5 to 1.0 commensurate monolayer can be attributed directly to the pressure shift of the melting transition.

\section{DISCUSSION}

The $\mathrm{N}_{2} / \mathrm{Ag}(111)$ system appears to be an intrinsic planar molecular solid and thus is an important member of the fam- ily of adsorbed molecular solids for establishing trends that depend on substrate corrugation. The inferred bulk modulus and the lattice constants of the observed orientationally disordered triangular lattice are in fair agreement with results of calculations for an interaction model with no corrugation but otherwise very similar to that used for $\mathrm{N}_{2} /$ graphite.

In a broader context, we have made quantitative predictions of phase transition temperatures starting from molecular models. The circumstances for $\mathrm{N}_{2} / \mathrm{Ag}(111)$, where corrugation effects appear to be small, should enhance the accuracy of the modeling. Two immediate tests are available. (1) We find an onset of 2-in herringbone ordering ${ }^{26}$ in a rectangular lattice distorted from a triangular center-of-mass lattice at temperatures a little below $20 \mathrm{~K}$. It would be of great interest to confirm the shift from the $26-28 \mathrm{~K}$ transition temperature of $\mathrm{N}_{2}$ /graphite and the change in lattice symmetry as orientational ordering transitions have not yet been observed in two closely related cases, $\mathrm{N}_{2} / \mathrm{Cu}(110)$ and $\mathrm{N}_{2} / \mathrm{MgO}(001)$. (2) We predict that melting of the $\mathrm{N}_{2} / \mathrm{Ag}(111)$ solid may be observed at temperatures of about $40 \mathrm{~K}$ if the 3D pressures can be held low enough that the unconstrained, nearly zero spreading pressure, solid is probed. Confirmation of the reduction in melting temperature from the $48 \mathrm{~K}$ of submonolayer $\mathrm{N}_{2}$ /graphite would provide support for the interpretation of incipient triple-point systems as having the solid preempting the liquid phase until the critical point is reached.

\section{ACKNOWLEDGMENTS}

This work has been partially supported by the National Science Foundation under Grant No. DMR-9423307 (L.W.B.) and by The Danish Natural Science Foundation (F.Y.H.). L.W.B. thanks the Department of Chemistry and the Technical University of Denmark for hospitality during the period this work was begun. We thank the authors of Refs. 3 and 7 for prepublication copies of their work.
${ }^{1}$ T. A. Scott, Phys. Rep. 27, 89 (1976) and references contained therein.

${ }^{2}$ L. W. Bruch, M. W. Cole, and E. Zaremba, Physical Adsorption: Forces and Phenomena (Oxford University Press, New York, 1997).

${ }^{3}$ G. S. Leatherman and R. D. Diehl, Langmuir 13, 7063 (1997).

${ }^{4}$ D. Marx and H. Wiechert, Adv. Chem. Phys. 95, 213 (1997).

${ }^{5}$ F. Y. Hansen, L. W. Bruch, and H. Taub, Phys. Rev. B 52, 8515 (1995).

${ }^{6}$ M. Trabelsi, J. P. Coulomb, D. Degenhardt, and H. Lauter, Surf. Sci. 377-379, 38 (1997).

${ }^{7}$ P. Zeppenfeld, J. Goerge, V. Diercks, R. Halmer, R. David, G. Comsa, A. Marmier, C. Ramseyer, and C. Girardet, Phys. Rev. Lett. 78, 1504 (1997); A. Marmier, C. Ramseyer, P. N. M. Hoang, C. Girardet, J. Goerge, P. Zeppenfeld, M. Büchel, R. David, and G. Comsa, Surf. Sci. 383, 321 (1997).

${ }^{8}$ F. Y. Hansen, L. W. Bruch, and H. Taub, Phys. Rev. B 54, 14077
(1996) and references contained therein.

${ }^{9}$ A. C. Kummel, G. O. Sitz, R. N. Zare, and J. C. Tully, J. Chem. Phys. 89, 6947 (1988).

${ }^{10}$ The calculated binding energy of $69 \mathrm{meV}$ reported in Ref. 9 was obtained by summing over only the topmost layer of $\mathrm{Ag}$ atoms; the corresponding value of $\omega_{\perp}$ is $4.4 \mathrm{meV}$. The sum is done for two layers in the present work in order to bring the monolayer energy closer to the experimental value (Ref. 3) of $103 \mathrm{meV}$ and thus to reduce the possibility of nonwetting introduced by an artificially weak substrate binding.

${ }^{11}$ F. Y. Hansen, V. L. P. Frank, H. Taub, L. W. Bruch, H. J. Lauter, and J. R. Dennison, Phys. Rev. Lett. 64, 764 (1990).

${ }^{12}$ J. Cui, D. R. Jung, and R. D. Diehl, Phys. Rev. B 45, 9375 (1992); R. Vollmer, Ph.D. thesis, University of Göttingen, 1991 (unpublished); K. D. Gibson and S. J. Sibener, J. Chem. Phys. 88, 7862 (1988).

${ }^{13}$ M. Gruyters and K. Jacobi, Chem. Phys. Lett. 225, 309 (1994). 
${ }^{14}$ W. A. Steele, Interaction of Gases with Solid Surfaces (Pergamon, Oxford, 1974).

${ }^{15}$ F. Y. Hansen and L. W. Bruch, Phys. Rev. B 51, 2515 (1995).

${ }^{16}$ S. E. Roosevelt and L. W. Bruch, Phys. Rev. B 41, 12236 (1990).

${ }^{17}$ M. P. Allen and D. J. Tildesley, Computer Simulation of Liquids (Oxford University Press, New York, 1987), Sec. 2.4; J. Alejandre, D. J. Tildesley, and G. A. Chapela, J. Chem. Phys. 102, 4574 (1995).

${ }^{18}$ For instance, for the $\mathrm{Ag}$ models at $15 \mathrm{~K}$, without the McLachlan term the triangular lattice with $L=4.16 \AA$ has $\phi_{x x}=-0.8$ and $\phi_{y y}=1.4 \mathrm{~K} / \AA^{2}$ and with that term, the triangular lattice with $L=4.21 \AA$ has $\phi_{x x}=-1.2$ and $\phi_{y y}=2.6 \mathrm{~K} / \AA^{2}$.

${ }^{19}$ For spherical adsorbates, see H.-Y. Kim and W. A. Steele, Phys. Rev. B 45, 6226 (1992).

${ }^{20}$ B. Kuchta and R. D. Etters, Phys. Rev. B 54, 12057 (1996).

${ }^{21}$ V. R. Bhethanabotla and W. A. Steele, Langmuir 3, 581 (1987).

${ }^{22}$ That the classical-limit quasiharmonic approximation gives a larger molar area than the molecular-dynamics result is probably a consequence of an incipient failure of the quasiharmonic approximation. The failure arises through an artificial dilation of the lattice, to the point of dynamical instability, to lower the free energy by lowering the harmonic frequencies. In this case, a local minimum in the free energy is still found at $20 \mathrm{~K}$ but not at $21 \mathrm{~K}$.
${ }^{23}$ T. Angot, Ph.D. Thesis, Université D’Aix Marseille II, 1991 (unpublished).

${ }^{24}$ M. T. Alkhafaji, P. Shrestha, and A. D. Migone, Phys. Rev. B 50, 11088 (1994); A. D. Migone (private communication).

${ }^{25}$ K. Morishige, K. Inoue, and K. Imai, Langmuir 12, 4889 (1996).

${ }^{26}$ This terminology follows A. B. Harris and A. J. Berlinsky, Can. J. Phys. 57, 1852 (1979). The 2-in herringbone is a two-sublattice structure with two glide planes and the molecular axes parallel to the adsorption plane.

${ }^{27}$ To estimate the reliability of the estimated transition temperature for $\mathrm{N}_{2} / \mathrm{Ag}(111)$, consider the results for commensurate $\mathrm{N}_{2}$ /graphite, where the transition is known to occur at 26-28 K. The methods were previously applied (Ref. 15) to the graphite $X 1 M$ case and gave a transition temperature of $22-23 \mathrm{~K}$. Repeating those calculations with the $X 1$ model gives very similar results. The uncertainty in determining the transition temperature is larger than the differences between the two sets of $S(1,2)$ data. Thus, the estimated transition temperature for $\mathrm{N}_{2} / \mathrm{Ag}(111)$ may be low by about $5 \mathrm{~K}$.

${ }^{28}$ Qualitative aspects of nitrogen on flat graphite were explored, with a different interaction model, by J. Talbot, D. J. Tildesley, and W. A. Steele, Mol. Phys. 51, 1331 (1984). In particular they found that a submonolayer patch on smooth graphite at $16 \mathrm{~K}$ dilates and distorts from the commensurate structure. 